\title{
A SUFFICIENT CONDITION THAT A MAPPING OF RIEMANNIAN MANIFOLDS BE A FIBRE BUNDLE
}

\author{
ROBERT HERMANN ${ }^{1}$
}

1. Introduction. All manifolds, maps, tensor-fields, curves, etc. will be of differentiability class $C^{\infty}$ unless mentioned otherwise. If $x$ is a point of a manifold $X, X_{x}$ denotes the tangent space at $x$. If $\phi: X \rightarrow B$ is a $C^{\infty}$ map of manifolds, $\phi_{*}: X_{x} \rightarrow B_{\phi(x)}$ is the linear map on tangent vectors induced by $\phi$. We suppose

(a) $\phi$ is of maximal rank on $X$, i.e. $\phi_{*}\left(X_{x}\right)=B_{\phi(x)}$ for all $x \in X$.

(b) $X$ and $B$ are Riemannian manifolds, and the isomorphism $\phi_{*}: X_{x} / \phi_{*}^{-1}(0) \approx B_{\phi(x)}$

preserves the inner-products defined by the metrics on these spaces, for all $x$ in $X$.

The main theorem is:

Theorem 1. If $X$ is complete as a Riemannian space, so is $B . \phi$ is then a locally trivial fibre space. If in addition the fibres of $\phi$ are totally geodesic submanifolds of $X, \phi$ is a fibre bundle with structure group the Lie group of isometries of the fibre.

2. Generalities [2;1]. If $x \in X, v_{1}, v_{2} \in X_{x}$, let $\left(v_{1}, v_{2}\right)$ denote the inner product that defines the Riemannian metric. If $\sigma:[0,1] \rightarrow X$ is a curve, let $\sigma^{\prime}: t \rightarrow \sigma^{\prime}(t)$ denote the tangent vector field to $\sigma$. If $v: t \rightarrow v(t) \in X_{\sigma(t)}$ is a vector-field along $\sigma$, let $\Delta v$ denote the covariant derivative of $v$ along $\sigma[1]$.

Let us recall how it is defined by Elie Cartan's method of orthonormal moving frames: Suppose $U$ is an open set of $X$ and $w_{i}(1 \leqq i, j, k \cdots \leqq n=\operatorname{dim} X$, summation convention) 1 -differential forms in $U$ with

$$
d s^{2}=w_{i} \cdot w_{i} \text { (Symmetric Product). }
$$

There are unique 1 -forms $w_{i j}$ in $U$, called the connection forms, such that

$$
\begin{aligned}
d w_{i} & =w_{i j} \wedge w_{j} \quad(\text { Exterior Product }), \\
w_{i j} & =-w_{j i} .
\end{aligned}
$$

Received by the editors April 10, 1959.

1 This work was supported in part by OOR, U. S. Army under contract No. DA 19020-ORD-3778. 
Then, for the portion of $\sigma$ in $U, \Delta v(t) \in X_{\sigma(t)}$ is defined by the condition:

$$
w_{i}(\Delta v(t))=\frac{d}{d t} w_{i}(v(t))-w_{i j}\left(\sigma^{\prime}(t)\right) w_{j}(v(t)) .
$$

Suppose now that $\sigma_{\mathrm{s}}$ is a one-parameter family of curves, a deformation of $\sigma$, i.e. $\sigma_{0}=\sigma$, and $v$ is the corresponding infinitesimal deformation field, i.e. $v(t)$ is the tangent vector to the curve $s \rightarrow \sigma_{s}(t)$ at $s=0$. Put $f(s)=$ length of the curve $\sigma_{s}$, and suppose each curve $\sigma_{s}$ is parametrized proportionally to arc-length. The formula for the "first variation" of arc length is then:

$$
\begin{aligned}
f^{\prime}(0) & =\int_{0}^{1}\left(\sigma^{\prime}(t), \Delta v(t)\right) / f(0) d t \\
& =\left(\sigma^{\prime}(t), v(t)\right) /\left.f(0)\right|_{t=0} ^{t=1}-\int_{0}^{1}\left(\Delta \sigma^{\prime}(t), v(t)\right) / f(0) d t .
\end{aligned}
$$

The condition that $\sigma$ be a geodesic is then

$$
\Delta \sigma^{\prime}(t)=0 .
$$

3. Proof of Theorem 1. Let us return now to the map $\phi: X \rightarrow B$ of $\S 1$. For $x \in X$, a $v \in X_{x}$ is said to be vertical if $\phi_{*}(v)=0$. The vectors in the orthogonal complement to the vertical vectors are called horizontal. $P_{V}$ and $P_{H}$ denote the projections of $X_{x}$ on these subspaces. The field $x \rightarrow P_{V}\left(X_{x}\right)$ of tangent subspaces defines, with the metric, a "bundle-like" foliation on $X$ [7]. A curve in $X$ is said to be horizontal if its tangent vector field is horizontal. If $\sigma:[0,1] \rightarrow B$ is a curve, $x_{0} \in \phi^{-1}(\sigma(0))$, there is at most one horizontal lifting $\sigma_{1}:[0,1]$ $\rightarrow X$ of $\sigma$ beginning at $x_{0}$, and horizontal liftings always exist locally [4]. If they always exist globally as well, the field of horizontal subspaces is a connection for the map $\phi$ in the sense of Ehresmann [4], and the map $\phi$ is a locally trivial fibre space.

Proposition 3.1. If $\sigma:[0,1] \rightarrow X$ is a curve, $v$ a vector field along $\sigma, \phi \sigma: t \rightarrow \phi \sigma(t)$ and $\phi_{*} v: t \rightarrow \phi_{*} v(t)$ the projections in $B$, then

$$
\Delta \phi_{*}(v)=\phi_{*}\left(\Delta\left(P_{H}(v)\right)\right) .
$$

Hence in particular the projection of a horizontal geodesic of $X$ is a geodesic of $B$, and conversely the horizontal lifting of a geodesic of $B$, if it exists, is a geodesic.

Proof. Suppose $\operatorname{dim} B=n-m$. Adopt the following ranges of in- 
dices and summation conventions: $1 \leqq a, b, c, \leqq m ; m+1 \leqq u, v, \cdots$ $\leqq n$. Let $\theta_{u}$ be 1 -forms in an open set $U$ of $B$ with

$$
d s^{2}=\theta_{u} \cdot \theta_{u} .
$$

Suppose $\theta_{u v}$ are the corresponding connection forms. Let $w_{i}$ be 1 -forms in an open set $U^{\prime}$ of $X$ with $\phi\left(U^{\prime}\right)=U$ and $d s^{2}=w_{i} \cdot w_{i}$, $w_{u}=$ the one form on $U^{\prime}$ induced by $\phi$ from $\theta_{u}$, to be denoted here by $\phi^{*}\left(\theta_{u}\right)$.

Suppose $\theta_{u v}=f_{u v w} \theta_{w}, w_{i j}=g_{i j k} w_{k}$, where $w_{i j}$ are the connection forms corresponding to $w_{i}$ in $U^{\prime}$. One derives by calculation:

$$
\begin{aligned}
& \text { (a) } g_{u v w}=\phi^{*}\left(f_{u v w}\right), \\
& \text { (b) } g_{u a v}=g_{u v a}, \\
& \text { (c) } g_{u a b}=g_{u b a .}
\end{aligned}
$$

(3.2a) now implies 3.1 , via 2.1 .

Proposition 3.2. If $X$ is complete as a Riemannian metric space, so is $B$. In this case the horizontal liftings of paths ${ }^{2}$ of $B$ exist globally, i.e. $\phi$ is a fibre space.

Proof. The horizontal lifting of a small geodesic segment of $B$ is a geodesic segment. If $X$ is complete it can be completely extended to a geodesic of infinite length. The projection in $B$ of the extension is an extension of the given segment to an infinite geodesic, hence every geodesic segment of $B$ can be indefinitely extended.

To prove the second part, note that the horizontal lifting of a path of $B$ has the same length. If $\sigma:[0,1] \rightarrow B$ is a path, local horizontal liftings always exist; if we try to get a global lifting by the usual process of continuation we run into no obstruction since the partial liftings will always lie in a fixed bounded, hence compact region of $X$.

We assume from now on that every path in $B$ has horizontal liftings starting at arbitrary points of the initial fibre. If $\sigma$ is a path: $[0,1] \rightarrow B$, there is a diffeomorphism $h_{\sigma}: \phi^{-1}(\sigma(0)) \rightarrow \phi^{-1}(\sigma(1))$ obtained by mapping each $x_{0} \in \phi^{-1}(\sigma(0))$ into the end-point of the horizontal lifting of $\sigma$ starting at $x_{0}[4]$.

Proposition 3.3. If all the fibres of $\phi$ are totally geodesic submanifolds of $X$, then for each path $\sigma$ of $B, h_{\sigma}$ is an isometry of $\phi^{-1}(\sigma(0))$ onto $\phi^{-1}(\sigma(1))$.

Proof. We use the first variation formula 2.2. Suppose $\alpha:[0,1] \rightarrow X$

${ }^{2}$ A path is a continuous image of $[0,1]$ that is composed of a finite number of unbroken $C^{\infty}$ curves. 
is a curve completely on a fibre of $\phi$, i.e. a vertical curve. Suppose we deform $\alpha$ in a horizontal direction i.e. $\alpha_{\varepsilon}$ is a one-parameter family of curves, with infinitesimal deformation field $v: t \rightarrow v(t) \in X_{\alpha(t)}$ a horizontal curve. If $f(s)=$ length of $\alpha_{\varepsilon}, 2.2$ says that $f^{\prime}(0)=0$, since $\Delta \alpha^{\prime}$ is a vertical vector field (because the fibre is totally geodesic).

If $\sigma:[0,1] \rightarrow B$ is a curve, the image curve of a curve in $\phi^{-1}(\sigma(0))$ by $h$ is obtained by deforming along horizontal curves covering $\sigma$, hence has the same length. Q.E.D.

We assume then that the fibres of $\phi$ are totally geodesic. Fix a $b_{0} \in B$, and set $F=\phi^{-1}\left(b_{0}\right)$. Let $G$ be the Lie group of isometries of $F$. If $b \in B$, let $G_{b}$ be the set of isometries of $F$ onto $\phi^{-1}(b)$. Let $E$ be the point-set union of all $G_{b}$, for $b \in B$. Let $p: E \rightarrow B$ be the map $G_{b} \rightarrow b$. $G$ acts in an obvious way on $E$ on the left, and $E$ would be a principal fibre bundle with $G$ as structure group, except that so far it has no topology or manifold structure. Suppose though one can find a locally finite open covering of $B,\{U\}$, such that in each open set $U$ of the covering there is a cross-section $f_{U}: U \rightarrow E$. Then, for each $U$ and $U^{\prime}$ of the covering that intersect, there is a map $g_{U U^{\prime}}: U \cap U^{\prime} \rightarrow G$ such that

$$
f_{U^{\prime}}(b)=g_{U^{\prime} U}(b) f_{U}(b) \quad \text { for } b \in U \cap U^{\prime} .
$$

Suppose that the covering and the cross-sections can be chosen so that all the maps $g_{U^{\prime} U}$ are $C^{\infty}$; clearly then they define a $C^{\infty}$ coordinate bundle in the sense of Steenrod [8] which is isomorphic to $E$, and one can use this isomorphism to define $E$ as a $C^{\infty}$ manifold.

We use Proposition 3.3 to define such cross-sections. Choose the covering so that each $U$ is a geodesically convex open set of $B$, and pick a point $b_{U}$ in $U$ and a curve $\sigma_{U}$ joining $b_{0}$ to each $b_{U}$. Any $b \in U$ is on a unique geodesic with $b_{U}$, and one obtains a path $\sigma_{b, U}$ depending on $b$ and $U$ joining $b_{0}$ with $b$. Define $f_{U}(b)=h_{\sigma b, U} \in B_{b}$. It is easy to see that the transition functions $g_{U U^{\prime}} \rightarrow G$ are $C^{\infty}$; they are defined by solutions of $C^{\infty}$ ordinary differential equations depending in a $C^{\infty}$ way on initial conditions.

Having defined $E$ as a $C^{\infty}$ principal fibre bundle with structure group $G$, it is clear that the given fibre space $\phi: X \rightarrow B$ is isomorphic to the associated bundle with fibre $F$. The proof of Theorem 1 is then finished.

Let $\Omega$ be the space of loops of $B$ starting at $b_{0} . h: \sigma \rightarrow h_{\sigma}$ is then a map $\Omega \rightarrow G$. Let $B_{G}$ be the base of the universal classifying bundle for $G$. The bundle $E$ is induced by a map, the classifying map, $B \rightarrow B_{G}$. The loops of $B_{G}$ have the same homotopy type as $G$, and it is clear that, up to homotopy, $h$ is the same as the map $\Omega \rightarrow \Omega\left(B_{G}\right)$ induced by the classifying map [6]. 
4. Some examples. First, note that the homogeneous spaces of Lie groups with compact isotropy groups provide a class of examples satisfying the hypotheses of Theorem 1 . Of course, Theorem 1 is well-known for these examples [8]. This suggests as generalization the mappings $\phi: X \rightarrow B$ which locally have the structure of principal fibre bundle with a Cartan connection [5]. Recall that the bundle of orthonormal frames of a Riemannian manifold has this sort of structure.

To describe these examples, one should be given

(1) a map $\phi: X \rightarrow B$ of manifolds which is of maximal rank everywhere, with $\operatorname{dim} X=n, \operatorname{dim} B=n-m$,

(2) a real Lie algebra of dimension $n, G$, a subalgebra $K$ of dimension $m$, a subspace $M$ of $G$ with $G=K \oplus M,[K, M] \subset M$, a positive definite bilinear form on $\boldsymbol{M}$ with respect to which the operations of Ad $K$ are skew-symmetric. (We follow Chevalley [3] for the notations of Lie algebra theory.) Choose a basis $\xi_{i}$ of $G$ such that (a) the $\boldsymbol{g}_{a}$ span $\boldsymbol{K}$ and the $\boldsymbol{g}_{u}$ span $\boldsymbol{M}$ (same range of indices as in $\S 3$ ), (b) the $\boldsymbol{g}_{u}$ are an orthonormal basis with respect to the bilinear form on $\boldsymbol{M}$. Let $\left[\boldsymbol{g}_{i}, \boldsymbol{g}_{j}\right]=c_{i j k} \boldsymbol{g}_{k}$ be the structural equations of $G$ with respect to this basis.

(3) $n$ vector-fields $\theta_{i}$ for $X$ which are linearly independent at every point of $X$, i.e. form a parallelism for $X$, such that

(a) $\left[\theta_{a}, \theta_{i}\right]=c_{a i j} \theta_{j}$, i.e. only part of the structural equations are satisfied, and

(b) for $x \in X$, the $\theta_{a}(x)$ form a basis for the vectors tangent to the fibre of $\phi$ through $x$.

One then defines a Riemannian metric for $X$ by the requirement that, for $x \in X$, the $\theta_{i}(x)$ form an orthonormal basis for $X_{x}$, i.e. if $w_{i}$ are the dual basis of differential forms of $X, w_{i}\left(\theta_{j}\right)=\delta_{i j}$, then

$$
d s^{2}=w_{i} \cdot w_{i} .
$$

Call the vectors spanned by the $\theta_{a}$ the vertical vectors and let $P_{H}$ and $P_{V}$ be the projections defined on $X_{x}$, as before.

Proposition 4.1. With this metric for $X$, if the fibres of $\phi$ are connected $B$ inherits a Riemannian metric such that, for each $x$, the isomorphism $\phi_{*}: P_{H}\left(X_{x}\right) \approx B_{\phi(x)}$ preserves inner products and the fibres of $\phi$ are totally geodesic.

Proof. Suppose $\left[\theta_{i}, \theta_{j}\right]=f_{i j k} \theta_{k}$, where the $f_{i j k}$ are real-valued $C^{\infty}$ functions on $X, f_{a j k}=c_{a j k}$. Then [3, p. 154]

$$
d w_{i}=\frac{1}{2} f_{j k i} w_{j} \wedge w_{k} .
$$


Suppose $w_{i j}=h_{i j k} w_{k}$ are the connection forms for the Riemannian metric, i.e. $d w_{i}=w_{i j} \wedge w_{j}, w_{i j}=-w_{j i}$.

One calculates:

$$
h_{i j k}=\frac{1}{2}\left(f_{k j i}-f_{j i k}+f_{i k j}\right)
$$

Then, if $\sigma:[0,1] \rightarrow K$ is a curve, and $v: t \rightarrow v(t) \in X_{\sigma(t)}$ is a vector field along $\sigma$,

$$
w_{i}(\Delta v(t))=\frac{d}{d t} w_{i}(v(t))-h_{i j k}\left(\sigma^{\prime}(t)\right) w_{k}\left(\sigma^{\prime}(t)\right) w_{j}(v(t)) .
$$

Now, suppose

$$
S:(s, t) \rightarrow S(s, t), \quad 0 \leqq s \leqq 1,0 \leqq t \leqq 1
$$

is a surface in $X$ such that: for fixed $s, t \rightarrow S(s, t)$ is vertical, i.e. lies in a fibre, and for fixed $t, s \rightarrow S(s, t)$ is horizontal.

Suppose $S^{*}\left(w_{i}\right)=A_{i}(s, t) d t+B_{i}(s, t) d s$. The geometric interpretation of these functions is that $A_{i} \theta_{i}(S(s, t))$ resp. $B_{i} \theta_{i}(S(s, t))$ is the tangent vector to the curve $t \rightarrow S(s, t)$ resp. $s \rightarrow S(s, t)$. Then

$$
A_{u}=0, \quad B_{a}=0 \text {. }
$$

From 5.1 we derive

$$
\frac{\partial}{\partial s} A_{i}-\frac{\partial}{\partial t} B_{i}=f_{j k i}(S(s, t)) B_{j} A_{k}
$$

To prove that $B$ inherits a Riemannian metric from the given metric on $X$, it suffices to show that, for every such surface $S$,

$$
\frac{\partial}{\partial t} B_{u} B_{u}=0
$$

for then it would follow that the lengths of all horizontal liftings of a given tangent vector to $B$ have the same length.

Using (4.4) and (4.5), we have

$$
\frac{\partial}{\partial t} B_{u} B_{u}=2 B_{u} c_{a u v} B_{v} A_{a}
$$

For each $a$, the matrix $c_{a v u}$ is the matrix of Ad $\boldsymbol{g}_{a}$ acting on $\boldsymbol{M}$ : by our assumption it is skew-symmetric, hence

$$
B_{u} c_{a v u} B_{v}=0 \text {. This proves (4.6). }
$$

We want to now show that the fibres of $\phi$ are totally geodesic. Let 
then $\sigma:[0,1] \rightarrow X$ be a curve in the fibre, i.e. $\sigma^{\prime}(t)$ is vertical, and $v: t \rightarrow v(t) \in X_{\sigma(t)}$ a vertical vector field along $\sigma$, i.e. $w_{u}(v(t))=0$. From (4.3) we have

$$
w_{u}(\Delta v)=-h_{u a b}(\sigma(t)) w_{b}\left(\sigma^{\prime \prime}(t)\right) w_{a}(v(t)) .
$$

From 4.2

$$
h_{u a b}=\frac{1}{2}\left(c_{b a u}-c_{a u b}+c_{u b a}\right) .
$$

That each of these three terms is zero follows from the relations $[K, K] \subset K,[K, M] \subset M$.

\section{BIBLIOGRAPHY}

1. R. Bott, An application of the Morse theory to the topology of Lie groups, Bull. Soc. Math. France vol. 4 (1956) pp. 251-281.

2. E. Cartan, Géométrie des espaces de Riemann, Paris, Gauthier-Villars, 1946.

3. C. Chevalley, Theory of Lie groups, I, Princeton, 1946.

4. C. Ehresmann, Les connexions infinitésimales dans un espace fibré différentiable, Colloque de Topologie, Bruxelles, 1950, pp. 29-55.

5. S. Kobayashi, On connections of Cartan, Canad. J. Math. vol. 8 (1956) pp. 145156.

6. R. Lashof, Classification of fibre bundles by the loop space of the base, Ann. of Math. vol. 64 (1956) pp. 436-446.

7. B. Reinhart, Foliated manifolds with bundle-like metrics, Ann. of Math. vol. 69 (1959) pp. 119-131.

8. N. Steenrod, Topology of fibre bundles, Princeton Mathematical Series, vol. 14, Princeton, 1951.

HARVARD UNIVERSITY 